\title{
Regulation of Carotenogenesis by Inorganic Phosphate in Blakeslea trispora
}

\author{
By J. N. DHOLAKIA*† AND V. V. MODI \\ Department of Microbiology, Faculty of Science, M. S. University of Baroda, \\ Baroda 390 002, India
}

(Received 20 December 1983; revised 6 March 1984)

\begin{abstract}
Increasing the inorganic phosphate concentration of the medium from $0.01 \%$ to $1 \%(\mathrm{w} / \mathrm{v})$ resulted in 4.5-fold higher production of carotene in Blakeslea trispora. The pattern of $\beta$-carotene synthesis in $B$. trispora remained essentially the same in both high $(1 \%)$ and low $(0.01 \%)$ phosphate grown cultures. Higher concentrations of intracellular orthophosphate and lower activities of acid and alkaline phosphatase were found in the high phosphate grown cells as compared to the low phosphate grown cultures. The intracellular acid and alkaline phosphatase activities of $\boldsymbol{B}$. trispora were competitively inhibited by inorganic phosphate. One or more forms of the acid and the alkaline phosphatases were apparently repressed during active carotenogenesis in the high phosphate grown $B$. trispora as compared to low phosphate grown cells. An inverse relationship was found between carotenogenesis and the intracellular phosphatase activities and it is suggested that inorganic phosphate concentration affects $\beta$ carotene synthesis in $B$. trispora by regulating the intracellular phosphatase levels in the culture.
\end{abstract}

\section{INTRODUCTION}

$\beta$-Carotene, a vitamin A precursor, is synthesized by a variety of micro-organisms. Blakeslea trispora displays one of the highest carotenogenic powers and synthesizes predominantly trans- $\beta$ carotene, the most potent biological form (Ciegler, 1965). It has been suggested that phosphate exerts a direct control on the selection of primary and secondary metabolism of the cell (Martin, 1977). The effect of phosphate in secondary metabolism and the mechanisms governing the yields of secondary metabolites have been reviewed by Demain (1972), Martin (1977) and Weinberg (1978). In general, the synthesis of various secondary metabolites, viz. antibiotics, alkaloids, prodigiosin, candicidin, aflatoxin, etc., is suppressed in medium high in phosphate but is stimulated with phosphate concentrations sub-optimal for the growth of the microorganism (Demain, 1972; Weinberg, 1974). Although the potential of $B$. trispora has been well recognized in industrial microbial $\beta$-carotene production (Ciegler, 1965), the regulation of carotenogenesis in $\boldsymbol{B}$. trispora by inorganic phosphate concentration of the medium has not been studied. In the present investigation we have studied the influence of inorganic phosphate on carotenogenesis in $\boldsymbol{B}$. trispora and an attempt has been made to correlate it with intracellular phosphatase activities.

\section{METHODS}

Culture and growth conditions. The plus (NRRL 2895) and minus (NRRL 2896) strains of Blakeslea trispora were obtained from the US Department of Agriculture, Illinois, USA, and were maintained as described earlier (Dandekar \& Modi, 1980). Mated cultures (equal volumes of plus and minus strains grown together) were used in all the experiments.

The synthetic medium employed for the growth of the fungus was the same as described earlier (Dholakia \& Modi, 1982). $\mathrm{KH}_{2} \mathrm{PO}_{4}$ was the source of inorganic phosphate. 'Low phosphate' indicates the addition of $0.01 \%$

† Present address: Department of Biochemistry, The University of Mississippi Medical Center, 2500 North State Street, Jackson, MS 39216, USA. 
(w/v) $\mathrm{KH}_{2} \mathrm{PO}_{4}$, while 'high phosphate' means the addition of $1.0 \%$ (w/v) $\mathrm{KH}_{2} \mathrm{PO}_{4}$ to a synthetic medium devoid of phosphate.

The method of Ciegler et al. (1962) was followed for the preparation of inoculum and a $10 \mathrm{ml}$ fraction of the mycelial homogenate was used to inoculate each of the flasks containing $100 \mathrm{ml}$ medium. Cultures were incubated at $28{ }^{\circ} \mathrm{C}$ on a gyratory shaker $(200$ r.p.m.) for $5 \mathrm{~d}$ unless specified in the experiment. Growth determination, and extraction and estimation of $\beta$-carotene were as reported by Dandekar \& Modi (1980).

Preparation of cell-free extract. Mycelia were harvested by filtration and washed with distilled water. A $30 \%$ (w/v) extract was prepared in $0.01 \mathrm{M}-\mathrm{Tris} / \mathrm{HCl}$ buffer $(\mathrm{pH} 7 \cdot 2)$ by grinding the frozen mycelia with Pyrex glass powder $(2: 1, w / w)$ using an unglazed mortar and pestle between 0 and $3{ }^{\circ} \mathrm{C}$, until complete cell breakage occurred (checked microscopically). The extract was then centrifuged at $15000 \mathrm{~g}$ for $20 \mathrm{~min}$ and the supernatant filtered through double layers of cheesecloth to remove floating scum usually present in the extract. The supernatant was used as the cell-free extract for the estimation of intracellular enzyme activities. To determine the initial reaction velocity of phosphatases, the cell-free extract was dialysed against $10 \mathrm{~mm}$-Tris/ $\mathrm{HCl}$ buffer for $24 \mathrm{~h}$ at $4{ }^{\circ} \mathrm{C}$ and was then used for the enzyme assays.

Protein determination. Protein was estimated by the Lowry method using bovine serum albumin (Sigma) as the standard protein.

Phosphate determination. Inorganic phosphate was estimated by the method of Fiske \& Subba Row (1925).

Enzyme assays. A method described by Torriani (1960) was followed to determine the acid and the alkaline phosphatase activities. In a typical assay, $2.5 \mathrm{~mm}$-p-nitrophenyl phosphate, $1 \mathrm{~mm}$-magnesium acetate, an appropriately diluted extract and $200 \mu \mathrm{mol}$ buffer in a total volume of $4 \mathrm{ml}$ were incubated at $30^{\circ} \mathrm{C}$ for $1 \mathrm{~h}$. For acid phosphatase determination, acetate buffer, pH 5.0, was used, and for alkaline phosphatase determination Tris/ $\mathrm{HCl}$ buffer, $\mathrm{pH} 8 \cdot 5$, was used. The reaction was terminated by adding $2 \mathrm{ml} 1 \mathrm{M}-\mathrm{Na}_{2} \mathrm{CO}_{3}$ and the amount of $p$ nitrophenol released was estimated spectrophotometrically at $410 \mathrm{~nm}$. One unit of acid or alkaline phosphatase is defined as the amount of enzyme which catalyses the liberation of $1 \mu \mathrm{mol} p$-nitrophenol in $1 \mathrm{~min}$ at $30^{\circ} \mathrm{C}$.

Gel electrophoresis. Polyacrylamide gel electrophoresis was performed according to the procedure of Davies (1964). The cell-free extract containing $100 \mu \mathrm{g}$ protein was applied to the top of a $7.5 \%(\mathrm{w} / \mathrm{v})$ polyacrylamide gel just before electrophoresis. The current applied was $5 \mathrm{~mA}$ per gel and the electrophoresis was completed at $5{ }^{\circ} \mathrm{C}$ in $75 \mathrm{~min}$. Bromophenol blue was used as the reference point of migration.

For detection of acid phosphatase (acetate buffer, pH 5.0) and alkaline phosphatase (Tris/HCl buffer, $\mathrm{pH} \mathrm{8.5)}$ activities on the gel, the method described by Mattoo \& Shah (1974) was followed. The gels were incubated at $30^{\circ} \mathrm{C}$ with a reaction mixture containing $0.2 \mathrm{M}$-buffer, $0.6 \mathrm{~mm}$-lead acetate, $1 \mathrm{~mm}$-magnesium acetate and $10 \mathrm{~mm}$ sodium $\beta$-glycerophosphate. At the end of the incubation $(4-6 \mathrm{~h})$ the gels were first washed in distilled water and then in $7 \%(\mathrm{v} / \mathrm{v})$ acetic acid for $2 \mathrm{~min}$, and finally immersed in a $1 \%(\mathrm{w} / \mathrm{v})$ solution of sodium sulphide for $5 \mathrm{~min}$. The bands of enzyme activity turned black against a faint yellow to white background.

Each value expressed is the average of three individual experiments that differed by less than $10 \%$.

Chemicals. $p$-Nitrophenyl phosphate, $p$-nitrophenol and sodium- $\beta$-glycerophosphate were from Sigma. All the other chemicals and reagents used were of analytical grade.

\section{RESULTS}

Increasing the inorganic phosphate concentration in the medium from $0.01 \%$ to $1 \%(\mathrm{w} / \mathrm{v})$ increased the $\beta$-carotene yield per $100 \mathrm{ml}$ medium by 4.65 -fold without affecting the growth of $B$. trispora (Fig. 1), hence the increased yield of $\beta$-carotene reflects the higher carotenogenic efficiency of $B$. trispora grown in medium containing a higher phosphate concentration. The specific $\beta$-carotene production ( $\beta$-carotene per $\mathrm{g}$ mycelial dry wt) was $\mathbf{4} \cdot 5$-fold higher in high phosphate $(1 \%, w / v)$ medium as compared to cells grown with low phosphate $(0.01 \%, \mathrm{w} / \mathrm{v})$. Inorganic phosphate concentration higher than $1 \%$ adversely affected both $\beta$-carotene synthesis and the growth of the culture. An increase in the final $\mathrm{pH}$ of the medium was noted with an increase in the inorganic phosphate concentration of the medium. The growth of the fungus was similar on both high and low phosphate media and carotenogenesis started on the third day in both cases (Fig. 2). However, $\beta$-carotene synthesis in $\boldsymbol{B}$. trispora grown in high phosphate medium was higher from the beginning and increased as the fermentation progressed. $\beta$ Carotene produced on the 5 th day of incubation in low phosphate grown cells was about $23 \%$ of the amount synthesized by a high phosphate grown culture. No change in the $\mathrm{pH}$ of the high phosphate medium was registered during the fermentation period, while the $\mathrm{pH}$ of the medium with low phosphate decreased as the fermentation progressed.

A time course study was conducted for the extracellular and intracellular inorganic phosphate 


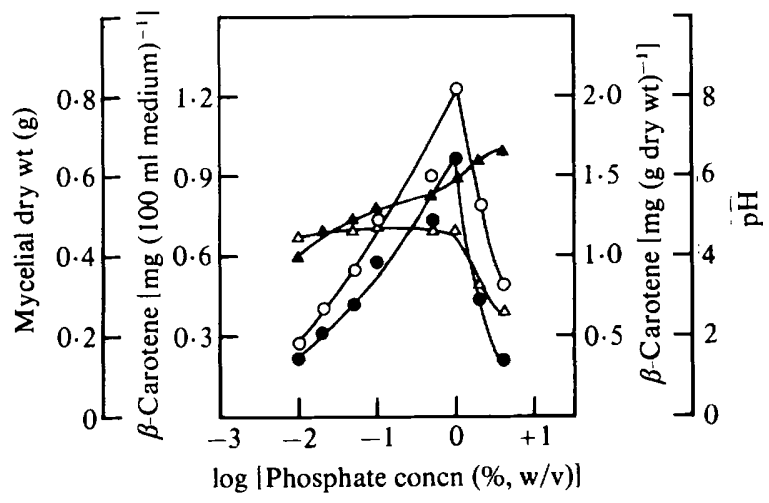

Fig. 1

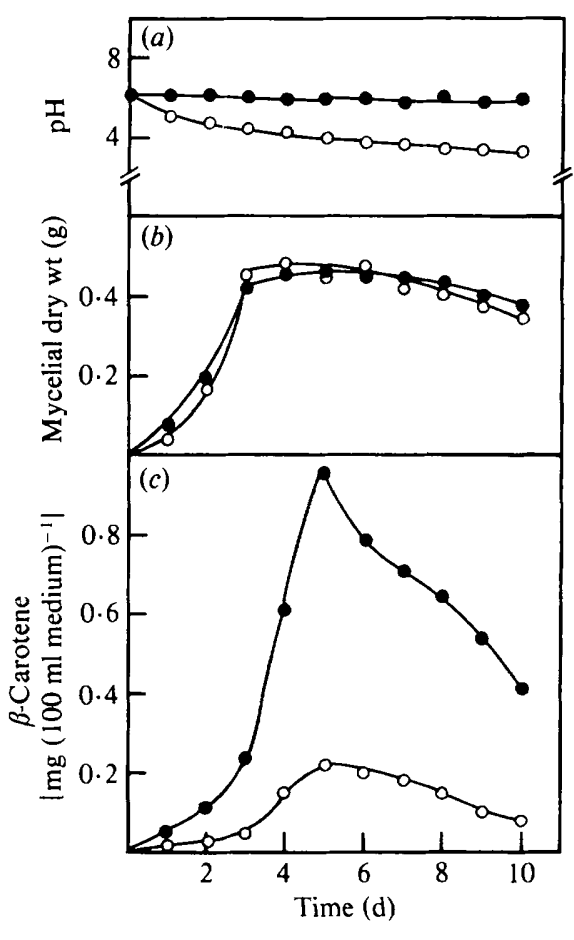

Fig. 2

Fig. 1. Effect of various concentrations of phosphate on the growth $(\Delta)$, final pH of the medium $(\Delta)$, $\beta$-carotene yields per $100 \mathrm{ml}$ medium $(O)$ and $\beta$-carotene synthesis per g mycelial dry wt $(O)$ in glucosegrown $B$. trispora.

Fig. 2. Time course of $(a)$ change in $\mathrm{pH}$ of the medium, $(b)$ growth and $(c) \beta$-carotene yields in high (O) and low $(O)$ phosphate grown $B$. trispora. High phosphate, $1 \%(w / v) \mathrm{KH}_{2} \mathrm{PO}_{4}$; low phosphate, $0.01 \%$ (w/v) $\mathrm{KH}_{2} \mathrm{PO}_{4}$.

concentration and phosphatase activity of $B$. trispora grown in high and low phosphate in order to find out the correlations, if any, between the phosphate concentration, carotenogenesis and phosphatase activity. The concentrations of extracellular and intracellular soluble phosphate during $\beta$-carotene fermentation are presented in Table 1 and Table 2, respectively. Considerable amounts of inorganic phosphate were present in the medium in the high phosphate grown cultures even after completion of fermentation, while in the low phosphate medium the extracellular phosphate was exhausted within $3 \mathrm{~d}$ of incubation. Correspondingly, the intracellular concentration of inorganic phosphate was much higher in high phosphate grown $B$. trispora as compared to low phosphate grown cells.

From Table 2 it is evident that both the acid and the alkaline phosphatase activities increased as fermentation progressed and thus followed the pattern of $\beta$-carotene production in $B$. trispora (see Fig. 2). In both the high and the low phosphate grown cells the alkaline phosphatase activity was higher than the acid phosphatase activity. The levels of phosphatases were found to be significantly lower throughout the fermentation cycle in the culture grown in high phosphate medium as compared to that grown in low phosphate.

The intracellular concentration of orthophosphate in the cells grown in the low phosphate medium decreased from 17.2 to $2.1 \mu \mathrm{mol}$ (g mycelial dry wt) ${ }^{-1}$, while there was a marked increase in the intracellular phosphatase activities with progress in the fermentation (Table 2). On the other hand, the intracellular concentration of orthophosphate remained above $17.2 \mu \mathrm{mol}$ ( $\mathrm{g}$ dry $\mathrm{wt})^{-1}$ in the high phosphate grown cells and the activity of phosphatases was very low. Thus the orthophosphate concentration in the high phosphate grown cells never reached the 
Table 1. Changes in the extracellular inorganic phosphate concentration due to the growth of B. trispora

\begin{tabular}{ccc}
$\begin{array}{c}\text { Time of } \\
\text { growth } \\
\text { (d) }\end{array}$ & \multicolumn{1}{c}{$\begin{array}{c}\text { Phosphate concn } \\
{\left[\mathrm{mg}(100 \mathrm{ml} \text { medium })^{-1}\right]}\end{array}$} \\
\cline { 2 - 3 } 0 & HP & LP \\
1 & $1000 \cdot 0$ & $10 \cdot 0$ \\
3 & $376 \cdot 7$ & $1 \cdot 27$ \\
5 & $252 \cdot 4$ & $0 \cdot 54$ \\
7 & $240 \cdot 1$ & $0 \cdot 24$ \\
9 & $225 \cdot 1$ & - \\
10 & $218 \cdot 5$ & -
\end{tabular}

*-, Not detectable; HP, high phosphate medium (1 $\mathrm{g} \mathrm{KH}_{2} \mathrm{PO}_{4}$ per $\left.100 \mathrm{ml}\right) ; \mathrm{LP}$, low phosphate medium $(0 \cdot 01 \mathrm{~g}$ $\mathrm{KH}_{2} \mathrm{PO}_{4}$ per $100 \mathrm{ml}$ ).

Table 2. Time course of intracellular acid and alkaline phosphatase activity and intracellular soluble inorganic phosphate concentration in $B$. trispora grown in high phosphate and low phosphate medium

High phosphate medium (HP) contained $1 \mathrm{~g} \mathrm{KH}_{2} \mathrm{PO}_{4}$ per $100 \mathrm{ml}$; low phosphate medium (LP) contained $0.01 \mathrm{~g} \mathrm{KH}_{2} \mathrm{PO}_{4}$ per $100 \mathrm{ml}$.

\begin{tabular}{|c|c|c|c|c|c|c|}
\hline \multirow{3}{*}{$\begin{array}{l}\text { Time of } \\
\text { growth } \\
\text { (d) }\end{array}$} & \multirow{2}{*}{\multicolumn{2}{|c|}{$\begin{array}{c}P_{1} \text { concn } \\
{\left[\mu \mathrm{mol}(\mathrm{g} \text { dry } w t)^{-1}\right]}\end{array}$}} & \multicolumn{4}{|c|}{ Phosphatase activity [units (g dry wt $)^{-1}$ ] } \\
\hline & & & \multicolumn{2}{|c|}{ Acid phosphatase } & \multicolumn{2}{|c|}{ Alkaline phosphatase } \\
\hline & HP & LP & HP & LP & HP & LP \\
\hline 1 & $69 \cdot 8$ & $17 \cdot 2$ & $2 \cdot 01$ & $9 \cdot 67$ & $9 \cdot 2$ & $29 \cdot 0$ \\
\hline 2 & $46 \cdot 3$ & $11 \cdot 8$ & $3 \cdot 21$ & $11 \cdot 68$ & $10 \cdot 5$ & $35 \cdot 1$ \\
\hline 3 & 32.9 & $6 \cdot 7$ & 4.67 & $16 \cdot 31$ & $13 \cdot 0$ & $48 \cdot 2$ \\
\hline 4 & $26 \cdot 4$ & $3 \cdot 4$ & $6 \cdot 33$ & $27 \cdot 70$ & $20 \cdot 4$ & $66 \cdot 5$ \\
\hline 5 & $19 \cdot 8$ & $2 \cdot 1$ & $8 \cdot 31$ & $31 \cdot 02$ & $28 \cdot 1$ & $76 \cdot 1$ \\
\hline
\end{tabular}

values of low phosphate cultivated cells. An inverse relationship between the intracellular concentration of orthophosphate and the activity of phosphatases was observed. Lower phosphatase activities in high phosphate grown $B$. trispora could be either due to feedback inhibition of the enzyme(s) by higher intracellular phosphate concentration and/or due to repression of phosphatases in high phosphate-containing medium.

A Lineweaver-Burk plot of the initial rate data of $p$-nitrophenyl phosphate (PNPP) hydrolysis obtained at various substrate concentrations in the absence and presence of $10^{-4} \mathrm{M}$-inorganic phosphate indicated that the inorganic phosphate competitively inhibited both the acid and the alkaline phosphatases from $B$. trispora. The $K_{\mathrm{i}}$ values for competitive inhibition of the enzyme by inorganic phosphate were $0.23 \mathrm{~mm}$ and $0.38 \mathrm{mM}$, while the $K_{\mathrm{m}}$ values (with respect to PNPP as substrate) were $0.11 \mathrm{~mm}$ and $0.29 \mathrm{mM}$ for the acid and the alkaline phosphatases, respectively. These results substantiate the view that the phosphatases of $B$. trispora are inhibited due to the higher intracellular phosphate concentration in the high phosphate grown cells.

The effect of the addition of $\mathrm{NaF}$, an inhibitor of phosphatases, to the synthetic medium on the levels of $\beta$-carotene (Fig. 3) and phosphatases (Fig. 4) was studied. As the earlier addition of $\mathrm{NaF}$ was found to alter the growth, $\mathrm{NaF}$ additions were made after $48 \mathrm{~h}$ and $72 \mathrm{~h}$ growth of the fungus. $\beta$-Carotene synthesis in low phosphate grown $B$. trispora was stimulated by the addition of $\mathrm{NaF}$ (Fig. 3) and it was accompanied by a considerable drop in the intracellular acid and alkaline phosphatase activity (Fig. 4). As compared to its addition after $72 \mathrm{~h}, \mathrm{NaF}$ addition after $48 \mathrm{~h}$ growth of the fungus was more effective in producing increased $\beta$-carotene yields and decreased phosphatase activity. This experiment clearly demonstrated an inverse relationship between carotenogenesis and intracellular phosphatase activities in $B$. trispora. 


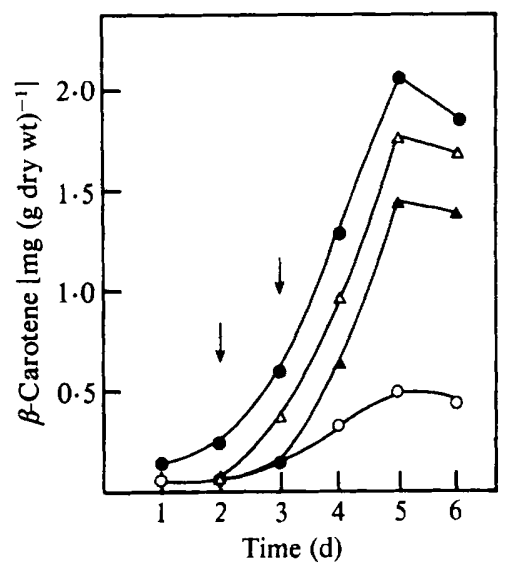

Fig. 3

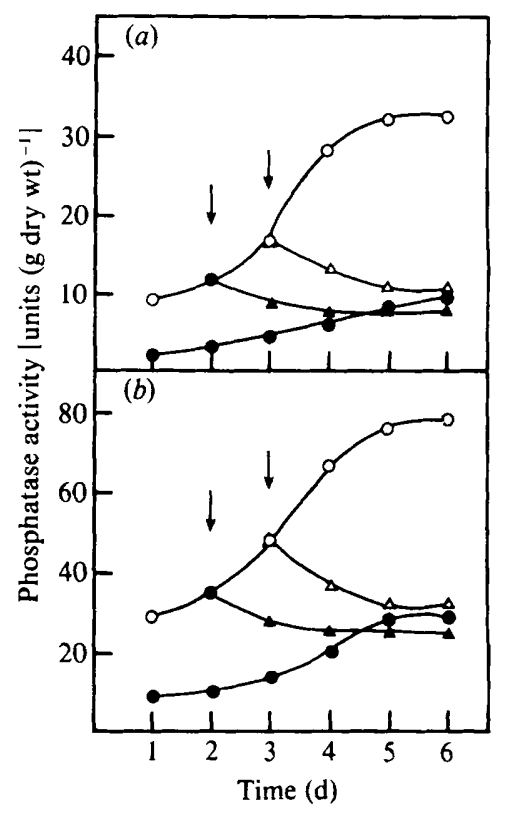

Fig. 4

Fig. 3. Effect of addition of $\mathrm{NaF}\left(1 \mu \mathrm{g} \mathrm{m} \mathrm{m}^{-1}\right)$ on carotenogenesis in low phosphate grown $B$. trispora. The arrows indicate the time of $\mathrm{NaF}$ addition. $\mathrm{O}$, Low phosphate (LP); $\triangle, \mathrm{LP}+\mathrm{NaF}(48 \mathrm{~h})$; $\Delta, \mathrm{LP}+\mathrm{NaF}(72 \mathrm{~h}) ; 0$, high phosphate.

Fig. 4. Effect of addition of $\mathrm{NaF}\left(1 \mu \mathrm{g} \mathrm{m}^{-1}\right)$ on $(a)$ intracellular acid phosphatase and $(b)$ intracellular alkaline phosphatase activities in $B$. trispora. The arrows indicate the time of $N a F$ addition. $O$, Low phosphate (LP); $\Delta, \mathrm{LP}+\mathrm{NaF}(48 \mathrm{~h}) ; \triangle, \mathrm{LP}+\mathrm{NaF}(72 \mathrm{~h}) ; \bullet$, high phosphate.

(a)

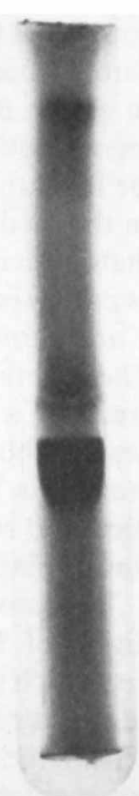

LP

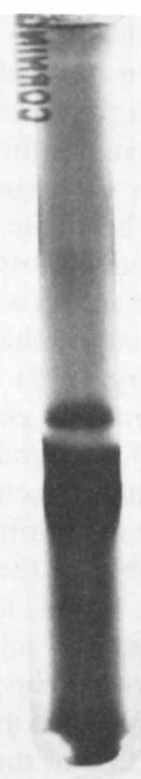

$\mathrm{HP}$ (b)

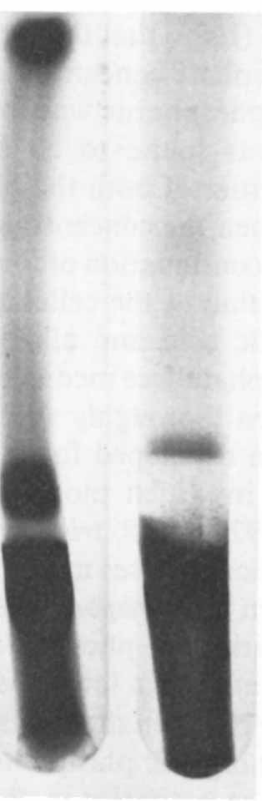

LP
HP

Fig. 5. Polyacrylamide gel electrophoretic pattern of $(a)$ intracellular acid phosphatase and (b) intracellular alkaline phosphatase from low phosphate (LP) and high phosphate (HP) grown B. trispora. 
The polyacrylamide gel electrophoretic pattern of the acid and the alkaline phosphatases from the cell-free extract of both the high and the low phosphate grown $B$. trispora was studied during the period of active carotenogenesis, i.e. 5th day of fermentation (Fig. 5). It is evident that one or more forms of the acid and the alkaline phosphatases was repressed in high phosphate grown cells as compared to low phosphate grown cells.

\section{DISCUSSION}

It has long been recognized that the synthesis of secondary metabolites has a much narrower tolerance for environmental concentrations of inorganic phosphate than does growth of the producer cells (Demain, 1972; Weinberg, 1974). The synthesis of various secondary metabolites was inhibited by high inorganic phosphate concentration of the medium (Weinberg, 1978). In the present study, we have found that unlike the general phenomenon of phosphate suppression of secondary metabolite formation, $\beta$-carotene production in $B$. trispora was stimulated by the higher phosphate concentration in the medium. The decrease in carotenogenesis at higher than $1 \%(\mathrm{w} / \mathrm{v})$ phosphate concentration could be due to inhibition of cellular metabolism by high inorganic phosphate concentration, as the growth of the culture was also reduced under these conditions. We had earlier found that the change in the final $\mathrm{pH}$ of the medium did not affect carotenogenesis in B. trispora (unpublished).

In a bleached strain of Euglena, low phosphate medium is reported to stimulate carotenogenesis threefold (Blum \& Begin-Heick, 1967), while in Ermothecium ashbyii, higher concentrations of inorganic phosphate were reported to increase flavin mononucleotide formation but decrease riboflavin synthesis (Mehta \& Modi, 1982).

One of the many possible mechanisms by which phosphate regulates the synthesis of a number of secondary metabolites is the repression or feedback inhibition of the enzymes releasing phosphate or pyrophosphate from phosphorylated intermediates by higher inorganic phosphate concentration of the medium (Martin, 1977). It has been reported that decrease in intracellular phosphatase activities resulted in increased carotenogenesis in Phycomyces blakesleeanus (Desai et al., 1973) and mango (Mangifera indica) fruits (Matoo et al., 1968).

Phosphate uptake is an energy-dependent process (Rosenberg et al., 1979) and it was reported by Ramasay \& Douglas (1979) that the intracellular phosphate content of the cells was directly proportional to the phosphate concentration in the growth medium. In our studies also, higher intracellular inorganic phosphorus was found in high phosphate grown $\boldsymbol{B}$. trispora and as the inorganic phosphate was found to be a competitive inhibitor of both acid and alkaline phosphatases, the activities of both the phosphatases were lower in B. trispora grown in high phosphate medium. When the concentration of the phosphate in the medium is very low, it is rapidly consumed. The continuation of metabolism under phosphate deficiency necessitates the turnover and redistribution of the cellular phosphate and this is catalysed by the activities of specific and non-specific acid and alkaline phosphatases that are known to be completely derepressed in the phosphate-free medium (Metzenberg, 1979). The genetics of the phosphatase enzyme system has been thoroughly studied in Neurospora crassa and a model based on the genetic results has been developed for the control of the synthesis of both acid and alkaline phosphatase enzymes, in which inorganic phosphate concentration is of great importance (Metzenberg \& Chia, 1979). In B. trispora both feedback inhibition and repression of both the acid and the alkaline phosphatases might be responsible for the lower phosphatase activities in high phosphate grown $B$. trispora which were found to be associated with higher carotenogenesis. Inhibition of phosphatase activities (by addition of $\mathrm{NaF}$ ) resulted in an increased $\beta$-carotene synthesis in low phosphate grown $B$. trispora, which further demonstrated the inverse relationship between the levels of carotenogenesis and the intracellular phosphatase activities. The role of inorganic phosphate concentration of the medium is thus to regulate the intracellular phosphatase activities in $B$. trispora, which in turn regulates the production of $\beta$-carotene.

Demain \& Inamine (1970) have reviewed Waksman's early suggestion that high inorganic phosphate concentrations suppress streptomycin formation by interfering with the production 
or action, or both, of one or more phosphatases involved in the synthesis of the antibiotic. A direct relationship between the alkaline phosphatase activity and neomycin formation has been reported in Streptomyces fradiae (Majumdar \& Majumdar, 1971).

Phosphatases are reported to affect the biosynthesis of sterols and lipids in various systems (Gore \& Popjak, 1962; Nightingale et al., 1967). Phosphatases were shown to limit the biosynthesis of squalene and sterol in neoplastic cells (Gore \& Popjack, 1962) and to be responsible for inhibiting the incorporation of acetate and mevalonate in sterols in cell-free preparations of testicular tissues (Nightingale et al., 1967). Acetate is the basic building block of the $\beta$-carotene molecule and various phosphorylated intermediates are required for the biosynthesis of $\beta$-carotene.

Our results will not only help in elucidating the regulatory mechanism of $\beta$-carotene synthesis but will also help in optimizing the medium constituents for an increased fermentative production.

\section{REFERENCES}

Blum, J. J. \& Begin-Heick, N. (1967). Metabolic changes during phosphate deprivation in Euglena in air and in oxygen. Biochemical Journal 105, 821-829.

Ciegler, A. (1965). Microbial carotenogenesis. Advances in Applied Microbiology 7, 1-34.

Ciegler, A., Nelson, G. E. N. \& Hall, H. H. (1962). Influence of hydrocarbon on carotenogenesis by mated cultures of Blakeslea trispora. Applied Microbiology 10, 132-136.

DANDEKaR, S. \& Modi, V. V. (1980). Involvement of cyclic AMP in carotenogenesis and cell differentiation in Blakeslea trispora. Biochimica et biophysica acta 628, 398-406.

DAvies, B. J. (1964). Disc electrophoresis. II. Method and application to human serum proteins. Annals of the New York Academy of Sciences 121, 404-427.

DEmain, A. L. (1972). Cellular and environmental factors affecting the synthesis and excretion of metabolites. Journal of Applied Chemistry and Biotechnology 22, 345-362.

Demain, A. L. \& InAmine, E. (1970). Biochemistry and regulation of streptomycin and mannosidostreptomycinase (alpha-D-mannosidase) formation. Bacteriological Reviews 34, 1-19.

Desai, H. G., Desai, J. D. \& Modi, V. V. (1973). Regulation of carotenogenesis in Phycomyces blakesleeanus. Indian Journal of Microbiology 13, 57-62.

Dholakia, J. N. \& Modi, V. V. (1982). Fermentative production of $\beta$-carotene and extracellular $\beta$-glucosidase by Blakeslea trispora grown on cellobiose. European Journal of Applied Microbiology and Biotechnology 15, 33-35.

Fiske, G. H. \& Subba Row, Y. (1925). The colorimetric determination of phosphorus. Journal of Biological Chemistry 66, 375-400.

GoRE, I. Y. \& PoPJAK, G. (1962). Sterol biosynthesis in neoplastic cells: utilization of ${ }^{14} \mathrm{C}$-acetate and ${ }^{14} \mathrm{C}$ mevalonate. Biochemical Journal 84, 93-99.

MaJumdar, M. K. \& MaJumdar, S. K. (1971). Relationship between alkaline phosphatase and neomycin formation in Streptomyces fradiae. Biochemical Journal 122, 397-404.
MARTIN, J. F. (1977). Control of antibiotic synthesis by phosphate. Advances in Biochemical Engineering 6, $105-127$.

Matoo, A. K. \& SнAH, Z. M. (1974). Nucleotide degrading enzymes in Neurospora crassa. Zeitschrift für allgemeine Mikrobiologie 14, 581-591.

Matoo, A. K., ModI, V. V. \& REDDY, V. V. R. (1968). Oxidation and carotenogenesis regulating factors in mangoes. Indian Journal of Biochemistry 5, 111-114.

MEHTA, H. B. \& MODI, V. V. (1982). Regulation of flavin levels by phosphate in flavinogenic Ermothecium ashbyii. Biochemistry International 2, 181-186.

MetzenberG, R. L. (1979). Implications of some genetic control mechanism in Neurospora. Microbiological Reviews 43, 361-383.

Metzenberg, R. L. \& ChiA, W. (1979). Genetic control of phosphorus assimilation in Neurospora crassa: dose-dependent dominance and recessiveness in constitutive mutants. Genetics 93, 625-643.

Nightingale, M. S., Tsai, S. C. \& Gaylor, J. L. (1967). Testicular sterols: VI. Incorporation of mevalonate into squalene and sterols by cell-free preparations of testicular tissue. Journal of Biological Chemistry 242, 341-349.

Ramasay, A. M. \& Douglas, L. J. (1979). Effects of phosphate limitation of growth on the cell wall and lipid composition of Saccharomyces cerevisiae. Journal of General Microbiology 110, 185-191.

Rosenberg, H., Gerdes, R. G. \& Harold, F. M. (1979). Energy coupling to the transport of inorganic phosphate in Escherichia coli K12. Biochemical Journal 178, 133-137.

ToRriani, A. (1960). Influence of inorganic phosphate in the formation of phosphatases in Escherichia coli. Biochimica et biophysica acta 38, 460-479.

WeINBERG, E. D. (1974). Secondary metabolism: control by temperature and inorganic phosphate. Developments in Industrial Microbiology 15, 70-81.

WeINBERG, E. D. (1978). Secondary metabolism: regulation by phosphate and trace elements. Folia microbiologica 23, 496-504. 